\title{
Guest Editorial to the Thematic Section on ECREA 2018
}

\author{
Katharina Lobinger, USI - Università della Svizzera italiana, Institute of Digital Technologies \\ for Communication (ITDxC) \\ Gabriele Balbi* , USI - Università della Svizzera italiana, Institute of Media and Journalism (IMeG) \\ Lorenzo Cantoni, USI - Università della Svizzera italiana Institute of Digital Technologies \\ for Communication (ITDxC) \\ *Corresponding author: gabriele.balbi@usi.ch
}

This thematic section is devoted to the $7^{\text {th }}$ Conference of the European Communication and Research Association (ECREA), held in Lugano from October $31^{\text {st }}$ to November $3^{\text {rd }}, 2018$. The section contains three different parts: three conference keynote speakers' papers translated into Italian language, six reports of special panels on topics related to the conference theme that were chosen by the local organizing committee of USI Università della Svizzera italiana, and, finally, a conference report written by Fiona Fehlmann. The reports on special panels are written in English language, the conference report in German and French language. This thematic section thus fully embraces the flexibility with respect to publication languages offered by Studies in Communication Sciences.

Why a thematic section dedicated to ECREA 2018? ECREA is the largest association of communication scholars in Europe and, as its $7^{\text {th }}$ general conference took place in Switzerland, it seemed important to bundle some discussions and reflections also in a Swiss academic journal in order to stimulate further conversations and debate. The aim of the conference was to address the theme "Centres and Peripheries: Communication, Research, Translation." This issue was addressed in several ways. In the following, three main lines of research and discussion that emerged will be taken up and discussed in more detail.

First, scholars have stretched the boundaries of media and communication research, going beyond the traditional "cores" and "margins" of this academic discipline. "Stretching" media and communication means bringing attention to under- developed fields of research and bringing theories, approaches and methods from other academic fields and disciplines into view. Academic subjects previously not concerned with aspects of mediated communication now acknowledge the role of media and communication processes for their discipline. Still, the role of "traditional" cores and centers might persist. Thus, changes and persistence provide a fascinating starting point for examining the identity and role of the discipline.

Second, the key conference theme of centers and peripheries particularly invited researchers to reconsider geographical, cultural and linguistic boundaries. Many areas of media and communication research have been "dominated" by American and European scholarship, which needs to be critically reflected. In this regard, as the subtitle of the conference emphasizing "translation" suggested, also a re-examination of the current dominance of the English language in academia is needed. There is no doubt that the English language has become the lingua franca of the scientific community, allowing scholars from different countries to communicate and to access each other's work. The Swiss plurilingual context provided a fruitful setting for revisiting and discussing the role of language in academia. Also, the present thematic section written in three languages can be considered a small linguistic experiment. But reconsidering borders also means engaging with key issues in communication studies, especially in the present global context of migration and mobility, where terms such as flow, mobility, multi-cultural, multi-perspec- 
tive, transcultural, hybrid and fragmented are ubiquitous.

Third, the key concepts of centers and peripheries in communication are associated with economic and political power. Communication flows often go from rich (central) countries to poor (peripheral) ones. Within single countries, distributions of resources are often unequal in terms of information and connectedness between privileged and unprivileged areas (e.g., urban peripheries and rural areas). People in disadvantaged areas are often excluded from flows, forms and practices of communication that are taken-for-granted in richer regions.

These and several other topics were discussed during four intense days in Lugano - a perfect venue to debate "center" and "periphery" because it is a city at the heart of Europe, geographically located near the borders of two countries, and it is the Italian speaking part and the southernmost region of Switzerland. The conference was extremely successful. During the submission process, we have received almost 2000 submissions from over 50 countries spanning five continents. After the reviewing process, more than 1100 presentations were accepted and presented. Overall, the conference had 1342 registered participants from all over the world. The top 10 countries per number of delegates were: Germany (215), United Kingdom (132), Switzerland (116), Spain (92), Denmark (87), Sweden (86), Netherlands (77), Belgium (62), Finland (56), and Portugal (52). Young and senior scholars from these countries and cultures created a sparkling and enriching environment, where European communication research was reconsidered.

\section{Translated articles by ECREA 2018 keynote speakers}

This variety of topics and countries was also mirrored by the talks of the four conference keynote speakers. In order of appearance, Eszter Hargittai (University of Zurich) gave a keynote speech on "The Online Participation Divide”, Lina Dencik
(Cardiff University) on "Resistance in the datafied society: from data ethics to data justice", José van Dijck (University of Utrecht) on "The Geopolitics Of Platforms: Lessons For Europe”, and finally Thomas Allmer (University of Stirling) launched the first ECREA Critical Intervention on "Academic Labour, Digital Media and Capitalism". In a tentative to bridge language boundaries, we invited the keynote speakers to identify one of their already published English papers related to the topics addressed in their keynote talks. These papers were then translated into Italian, the official language spoken in Lugano and at USI Università della Svizzera italiana, and for the first time they are now available to Italian speaking scholars and students. We hope they will be used in modules and courses, disseminating the important issues discussed there. As expected, also legal issues with translation arised. This is why, unfortunately, we did not obtain the permission of copyright holders to translate a paper by Eszter Hargittai.

The other three papers by keynote speakers published in this thematic section have at least two common features: in approaching communication research, they all adopt a critical perspective and they also intervene in contemporary debates inside and even outside academia. In José van Dijck's paper ("How to anchor public values in a connective world? Challenges for Europe"), the dominance of American and Chinese digital platforms is not only clearly illustrated and questioned, but five recommendations for $\mathrm{Eu}$ rope are provided to scholars, politicians, institutions, activists, and stakeholders. The main aim of these recommendations is to help Europe increase relevance in the global digital world. In "Surveillance Realism and the Politics of Imagination: Is There No Alternative?", Lina Dencik criticizes the contemporary surveillance culture and "dataism". According to her, data-driven surveillance is now considered "normal" and usually tolerated as if no alternatives existed, but this "surveillance realism" can be destroyed through an emancipatory politics of resistance and critique. Finally, Thomas Allmer in 
"I am a single mum. I don't feel like I can be as competitive as other people" shows how the economic, political and cultural transformations of British universities in the last decades might have produced insecurity, precariousness, overworked experiences, and might have even impacted mental and physical health of the academics. Strategies to overcome the challenges academics are facing today, he concludes, need to be found by politicians and university managers.

\section{ECREA 2018 Special Panel reports}

In the second part of this thematic section, you will find six special panel reports. As already described above, their themes were selected by the ECREA 2018 local organizing committee, who then approached expert researchers in the respective fields as panel organizers. Having special panels allowed us to give the heterogenous conference program a thematic focus that reflected the organizers ideas of the conference theme.

The first report by Sara Greco and Jolanta Drzewiecka presents the special panel entitled "Rediscussing centres and peripheries: communication lenses on migration". This panel focused on migration, understood as a poly-centric phenomenon that challenges the notions of center and periphery. One characteristic of this panel was, again, the critical focus, that stimulated a re-discussion of methods, approaches and starting points that often are taken for granted without being questioned. In the talks, particular emphasis was put on the role of data and of gathering data that are closely related to humanizing or dehumanizing practices.

The special panel "Re-booting gender in communication research and practice: a translational approach" was organized by Claudia Padovani and Karen Ross. Also in this panel, justice and equality were core themes and the panelists focused on the topic of gender equality and the role of representation, production, policy, and ownership dimensions. With the term "re-booting", the organizers particularly encouraged new ways of thinking and researching gender equality and the media, particularly inviting "translations" and "re-interpretations" of communication research findings.

Translation and language were also core topics of the third special panel presented in this issue. Helena Sousa put together a panel entitled "The English language in academia: Identifying power structures, denaturalizing daily choices". The panelists here critically reflected on the English language hegemony in academia, highlighting benefits, challenges and asymmetries caused by the choice of a seemingly natural lingua franca. That language-induced inequalities have received little attention, yet, was the starting point for discussions of this panel.

Joy Jenkins reports on the panel "Journalism from the peripheries. From the Swiss-Italian media system to other local dimensions" organized by Philip di Salvo. The panel focused on local journalism in less examined and thus "peripheral" areas, taking the Swiss-Italian media market as an example. The panel was also characterized by a dialogue among journalism researchers and journalists. Local journalism in the Swiss-Italian area was further complemented by international perspectives on other "peripheries", that is on other local and regional newspapers.

The subsequent panel report is concerned with the flow of people and the role of cultural heritage for putting centers and peripheries into contact. This special panel entitled "Communicating World Heritage to global audiences of travelers" was conceived and organized by Lorenzo Cantoni and Silvia De Ascaniis. In particular, the panelists focused on the role of Information and Communication Technologies for communicating heritage to global audiences of travelers. Emphasis was put on sustainability and responsibility in current tourism. These are necessary preconditions for protecting fragile tangible and intangible heritage.

The final report by Christian Schwarzenegger, Katharina Lobinger and Gabriele Balbi sums up the discussions and findings of the panel "Academic traditions in 
communication: Expanding the field and redrawing the boundaries". This panel provided the space for an academic introspection and reflections on the shifting centers, peripheries, and boundaries of the field. The fluidity or flexibility of the discipline holds many advantages but also comes with risks and fears. Terms such as "interdisciplinary no-man's land" point to the power struggles related to cores and centers of a discipline. While certainly not providing solutions, the panel provided an open setting for debating the identity and challenges of a living discipline in constant movement.

\section{Conference report}

The thematic section on ECREA 2018 ends with a conference report written by Fiona Fehlmann in German and French language. She reminds us that important academic topics notwithstanding, also coffee is not a peripheral dimension of a good conference. We hope that readers of SComS will enjoy this glimpse into some of the many topics of such a big European communication conference. 